\title{
L'exposition Espace mobile à la Galerie VoX, de Montréal : régénération esthétique de l'espace public aux marges du politique
}

The Espace Mobile Exhibition by Galerie VOX in Montreal: Aesthetic Expression and the Regeneration of Public Space

Jean-François Côté et Maude Pugliese

\section{OpenEdition \\ Journals}

Édition électronique

URL : http://journals.openedition.org/edc/730

DOI : 10.4000/edc.730

ISSN : 2101-0366

Éditeur

Université Lille-3

\section{Édition imprimée}

Date de publication : 1 décembre 2008

Pagination : 37-58

ISBN : 978-2-917562-00-0

ISSN : $1270-6841$

Référence électronique

Jean-François Côté et Maude Pugliese, « L'exposition Espace mobile à la Galerie VOX, de Montréal régénération esthétique de l'espace public aux marges du politique », Études de communication [En ligne], 31 | 2008, mis en ligne le 01 décembre 2010, consulté le 19 avril 2019. URL : http:// journals.openedition.org/edc/730 ; DOI : 10.4000/edc.730

Ce document a été généré automatiquement le 19 avril 2019

(C) Tous droits réservés 


\section{L'exposition Espace mobile à la Galerie VOX, de Montréal : régénération esthétique de l'espace public aux marges du politique}

The Espace Mobile Exhibition by Galerie VOX in Montreal: Aesthetic Expression and the Regeneration of Public Space

Jean-François Côté et Maude Pugliese

1 Le développement polymorphe des villes contemporaines, qui compte certainement aujourd'hui pour l'un des phénomènes universels les plus remarquables, s'accompagne étrangement d'un questionnement généralisé sur la manière dont il parvient à acquérir un sens précis et une signification déterminée; point d'aboutissement d'une logique d'urbanisation entamée dans le cours du mouvement large d'industrialisation dans le premier tiers du XIX ${ }^{e}$ siècle, il se poursuit aujourd'hui dans une ère postindustrielle dont la mondialisation donne apparemment la figure la plus tangible et la plus assurée. Et pourtant, au travers de ces repères historiques devenus pratiquement indiscutables, les façons d'habiter les villes échappent souvent aux représentations fidèles que l'on voudrait en donner, tiraillées qu'elles restent entre les faits, tout aussi bien établis par ailleurs, que sont l'anonymat des villes surgissant du conformisme urbain, la marginalisation de parties importantes des populations, ou les trajets touristiques accumulant les clichés au fil des parcours de ce que les villes veulent bien donner à voir. Spectacle du monde, oui, mais non moins travaillé par des tensions, des ambiguïtés et des contradictions qui, entre le spectaculaire et le vernaculaire de l'espace public des villes actuelles, fait apparaitre une circulation éminemment paradoxale ${ }^{1}$. Flâner, comme le proposait Baudelaire, ne correspond pas tout à fait au Zeitgeist des villes d'aujourd'hui, ou 
alors ne paraît pas être une activité reconnue dans la signification active que le poète lui prêtait.

C'est dans ce contexte que nous voudrions analyser ici l'initiative de la Galerie VoX, de Montréal, d'organiser une exposition-événement intitulée Espace mobile au printemps 2008; en invitant sept artistes à donner une forme spécifique à leur intervention de l'espace urbain montréalais, la Galerie VOX a permis de définir une expression inusitée de l'«habiter» en ville, dont elle a su tirer une expérience profondément originale, et génératrice d'une participation inouïe à l'espace public. L'expression artistique, ici, en prenant une forme s'imprégnant des tensions principales du contexte dans lequel elle était invitée à s'immerger, a permis de traduire au sein de l'espace public un « lieu mobile » d'interrogations redessinant les contours des marges du politique. Après avoir brièvement décrit les contenus et les formes de l'exposition Espace mobile, nous nous pencherons dans un second temps sur la compréhension de cette intervention en fonction du contexte contemporain des villes et de la participation artistique, afin de proposer, dans une troisième partie, une interprétation plus large des rapports entre esthétique et éthique sur le fond de cette problématique de l'espace public des villes contemporaines. Nous conclurons sur le rôle de régénération de l'espace public que traduit l'expression esthétique du rapport à la ville.

\section{Espace mobile à VOX : de l'expérience autre de la ville à la révélation de la ville « autre »}

3 En organisant l'exposition-événement Espace mobile au printemps 2008, les dirigeants de la Galerie VOX entraient directement en rapport avec une problématique de développement urbain qu'on pourrait dire exemplaire de certaines tendances actuelles de la mondialisation, qui font entre autres la promotion du tourisme de masse et qui tentent de structurer des initiatives explicites dans ce sens en réorganisant l'espace public. Le cas de Montréal reste sans doute à cet égard tout à fait particulier, en ce qu'il se présente sous l'appellation explicite $\mathrm{du}$ projet $\mathrm{du}$ Quartier des spectacles, vaste et ambitieux mouvement de rénovation urbaine visant le cœur du centre-ville de la métropole québécoise, et sa transformation en un lieu d'attractions nourri par l'abondance des festivals et d'activités culturelles dont la promotion (internationale en particulier) affiche, et voudrait même affirmer, jusqu'à la "nouvelle identité » de la ville ${ }^{2}$. Or curieusement, une telle initiative, qui appelle des investissements appréciables de fonds publics, et qui est à même de transformer un lieu au point de remodeler complètement sa fonction, n'a pas suscité depuis son lancement, en 2003, de grands débats politiques à Montréal - entièrement soutenu qu'il est, notamment, par l'actuelle administration du maire de la ville, Gérald Tremblay, ainsi que par d'importants intervenants tels Tourisme Montréal, Culture Montréal, et la Chambre de commerce locale (de même que par des gouvernements provincial et fédéral). C'est dans ce contexte de remodelage massif de l'espace urbain, dont les premiers exemples contemporains remontent au Paris de Haussmann, et dont Montréal a déjà aussi, notamment au moment de l'Expo 67, connu certains des effets restructurants, que se redéploie la vocation du nouveau Quartier des spectacles sur les lieux mêmes d'un secteur occupé jadis par le monde interlope (drogue, prostitution, shylocking, crime organisé, etc.) montréalais, et dont il récupère incidemment de manière opportuniste l'appellation de «Red light " ${ }^{3}$. C'est en fonction de ces paramètres à la fois très larges, puisqu'ils impliquent des responsabilités 
gouvernementales non seulement municipales, mais aussi provinciales et fédérales (voire une logique internationale), et très étroits, puisque l'on parle d'un espace urbain d'environ un kilomètre carré, que se définit la problématique du Quartier des spectacles, et l'exposition de la Galerie VOX ${ }^{4}$. À l'écart du clinquant qu'appelle le «spectaculaire » urbain contemporain (tel qu'analysé entre autres par Sharon Zukin ou Michael Sorkin), mais tout autant à l'écart du «vernaculaire » urbain dont les traces ne sont souvent perceptibles qu'au travers une attention soutenue à l'égard d'un milieu (comme le proposaient entre autres les travaux de Michel de Certeau, Luce Giard et Pierre Mayol), les interventions artistiques ont pris forme de différentes façons dans un rapport inventif à l'espace public urbain.

4 Ainsi, l'artiste James Partaik, dont le travail va de l'installation à la performance, a-t-il proposé des «tunnels sonores», à partir de trajets horizontaux, de «lignes transversales... dans l'espace public environnant VOX », offrant « la possibilité de circuler d'un espace à l'autre en passant à travers tous les obstacles... un peu à la manière d'une onde qui traverse la matière $»^{5}$. Rassemblement d'une expérience auditive formée par les fragments sonores de ces parcours tracés littéralement au travers de la matière urbaine, par des lignes droites dessinées en abstraction des tracés des rues et des immeubles rencontrés, dans laquelle se retrouvent autant des bruits de toutes sortes que des bruits "blancs", des éclats de conversation ou des moments de la routine quotidienne (intérieurs domestiques, HLM, etc.), des lieux hétéroclites (mosquée, centre commercial, boutique d'esthéticienne, poste de police, etc.), c'est tout un univers qui se révèle dans un condensé où l'expérience de la ville se donne comme dans la simultanéité de ses expressions diverses et multivoques.

Dans une forme se rapprochant davantage du « test » ou de l'« expérience in situ » que de l'observation de la pratique urbaine, les membres de L'atelier d'exploration urbaine SYNont quant à eux proposé « d'expérimenter ce patrimoine vivant, au moyen d'une action soulignant l'intensité circonstancielle et les possibilités de brassage social, d'usages et de micro-urbanité que le quartier recèle $»^{6}$. S'introduisant dans l'espace des pratiques et des circulations quotidiennes du quartier au moyen d'une table de billard, installée de façon temporaire dans une série d'endroits plus ou moins incongrus (sur l'esplanade de la Place des Arts, sur la rue, dans le Parc de la Paix, en plein cœur du Red light montréalais), les membres de SYN- ont proposé aux passants un cadre d'action inhabituel par rapport à celui généralement attribué à la rue comme espace fonctionnel de déplacement. Les passants invités à agir dans ce nouveau cadre, en entamant par exemple une partie de billard avec les membres de SYN- sur le site où la table en question était temporairement installée, ont pu y réaliser un potentiel insoupçonné d'investissement des lieux dans des règles de convenance, de jeux et de fonctionnalité remodelées. Expérience immédiate de la polysémie des espaces pour les uns, constatation « de la condition urbaine existante et [de] son développement potentiel » dans des contextes de transformation des codes pour les autres, c'est ainsi que l'intervention s'est déroulée ; les joueurs de billard urbains, ainsi que les spectateurs des enregistrements de ces parties projetés par la suite dans les locaux de VOX, ont pu entrevoir les possibilités de déphasage des espaces, sous un angle en quelque sorte parallèle à celui que recèlent les changements que subira cette portion de la ville, entre autres par la concrétisation du Quartier des spectacles, mais dans un sens tout autre évidemment que celui proposé dans ce contexte précis ${ }^{7}$.

Dans une direction d'investigation encore différente, Renaud Auguste-Dormeuil s'est penché dans le cadre d'Espace mobile (comme dans plusieurs autres de ses projets 
individuels, aux Musées des Beaux-arts d'Orléans, de Bourges, de Tours, de Chartres, et au Musée d'art Moderne de la Ville de Paris), sur « le souci de sécurité dans l'espace public des sociétés développées $»^{8}$. Selon une méthode cartographique, Auguste-Dormeuil relève, et révèle par là même, l'ensemble des dispositifs de sécurité, caméras de surveillance et autres technologies de protection, qui meublent discrètement l'espace urbain. En mettant en évidence au sein d'espaces publics divers les tracés en filigrane d'une structure de surveillance par laquelle on souhaite une "circulation sociale" contrôlée et sécurisée, l'installation d'Auguste-Dormeuil invitait à réfléchir sur le caractère subtil et souvent indécelable des mécanismes par lesquels est déterminée et maintenue une certaine homogénéité d'utilisation des lieux, de même que sur les enjeux, notamment économiques, sous-jacents à l'installation de tels mécanismes d'un panoptikon multipolaire tendant aujourd'hui à s'étendre et à se généraliser. Entre l'espace urbain démocratique des usagers et les stratégies du pouvoir qui l'encadrent, une tension habituellement impalpable surgit, qui renvoie à certains aspects des transformations subtiles des paramètres proprement politiques de la ville.

7 Les autres initiatives artistiques inscrites dans la programmation d'Espace mobile allaient également dans ce sens d'une interrogation plus poussée à l'égard de la ville et de ses expressions tangibles, ou possibles; ainsi de l'exploration des «non-lieux » temporaires où le «nomadisme » contemporain échoue, comme le documentait Jocelyn Robert, ou les captations vidéographiques aléatoires de Gilbert Boyer, véritable "film inconscient » de la texture urbaine, ou encore les « déambulations systématiques », et systématiquement reportées, de Christoph Fink, ou enfin, les interrogations "paysagistes» de Felicity Tayler, qui dans son projet Propagande picturale, reproduisait sur son chevalet des « vues de Montréal » issues d'une peintre du XIX ${ }^{\mathrm{e}}$ siècle, en suscitant du fait des discussions avec les passants9.

8 Dans son ensemble, l'exposition-événement Espace mobile proposait donc une interrogation sur les pratiques quotidiennes des lieux urbains en fragmentant tantôt leurs bruits, leurs structures visibles et temporelles, tantôt les codes qui entourent ces pratiques, tels qu'ils sont vécus immédiatement et tels qu'ils manifestent leur pouvoir régulateur. Il s'agissait de considérer ces fragments de la circulation urbaine à travers différents dispositifs, dont les objectifs étaient de les révéler dans un contexte externe à leur réalisation usuelle ${ }^{10}$. Véritable reconfiguration esthésique de l'expérience quotidienne de l'urbanité, révélation d'une ville autrement vécue et autrement possible, Espace mobile a proposé par le biais d'une esthétique du fragment une expression inédite des pratiques urbaines au sein d'un espace de communications publique sur la ville, souvent nourri seulement de discours compétitifs bien ficelés, à la composition interne hautement cohérente et thétique. Cette forme d'intervention dans l'espace public qu'Espace mobile a incarné d'une façon particulièrement évidente est partagée par plusieurs autres initiatives artistiques, autant à Montréal qu'ailleurs dans le monde. Pas nécessairement toujours nouvelle au plan esthétique, cette formule semble néanmoins vouloir s'insérer et prendre une place dans un espace de débats éthiques et politiques, comme en témoignent les diverses résonances d'Espace mobile. Afin de voir dans quelle mesure elle réussit à s'intégrer dans l'espace de production des représentations de la ville et quel efficace elle comporte pour la constitution d'un imaginaire collectif qui donne à la ville son sens et la possibilité d'être pratiquée, on peut situer cette forme d'intervention dans le contexte de la contribution de l'art au développement contemporain de la ville, et particulièrement dans le type de rapport qui se construit et se conçoit de cette façon. 


\section{Des villes en dérive...}

C'est apparemment le rôle qu'assument les artistes qui permet une telle déstabilisation des perceptions de l'univers urbain contemporain. Mais peut-être justement faut-il voir là aussi l'inverse : c'est-à-dire le questionnement qu'entretient l'expérience contemporaine de la ville, mais qui ne rejaillit dans l'espace public que de manière tronquée, ou trompeuse, dans cette espèce de mouvement pratiquement sourd et aveugle du "développement", associé si facilement à des phénomènes de mobilisation de masse (capitaux et individus confondus) liés aux transformations actuelles de la ville, sous l'impulsion de forces et de puissances relativement anonymes ; les artistes feraient alors jouer cette dynamique en eux, la révélant en quelque sorte "pour de vrai », et pour ce qu'elle est véritablement : une espèce de dérive, consentie initialement comme liberté de mouvement et d'autonomie, d'auto-création de la ville comme prérogative accordée par la modernité, mais orientée par des impératifs du moment, parfois anonymes, parfois sortis de nulle part, parfois même tout à fait improvisés. La ville-spectacle que l'on nous présente, sous ses aspects manifestement grossis, et dont témoigne si éloquemment un projet comme le Quartier des spectacles à Montréal, forge en effet une représentation qui montre cela et le masque, tout à la fois. Et les expressions artistiques prennent cette réprésentation à revers, révélant le masqué - sous la forme d'autres représentations.

10 Il y a quelque chose qui se joue dans l'espace public en milieu urbain en échappant à ces développements spectaculaires, et que seule l'attention reprise au travers de la geste esthétique permet apparemment de ressaisir : c'est l'espace d'indétermination de la ville, qui est également son espace de liberté et d'automonie, mais dont on voit bien qu'il est traversé de tensions entre des forces parvenant souvent à niveler la signification de cette liberté, ou à l'enchasser dans des paramètres étroits qui prétendent donner un sens à la ville et résoudre les significations conflictuelles qui règnent en son sein. Dans cette perspective, par exemple, la marginalisation de certaines parties des populations et des expressions les confine finalement au silence et à l'invisibilité, et un des motifs de l'exposition Espace mobile était bel et bien de faire accéder quelques-unes de ces expressions marginales au niveau de la représentation - soit de les faire voir et entendre, mais différemment, c'est-à-dire avec d'autres moyens qu'elles se donnent habituellement. Ainsi également des discussions suscitées par les artistes au fil de leurs projets avec les habitants du quartier, ou encore des réactions des différents publics visitant la Galerie VOX et prenant contact avec le quartier, et avec la ville, d'une façon autre ${ }^{11}$. Étrangement, ainsi, s'établit un rapport à la ville dans un espace public qui réapparaît au travers de la plasticité dont il est pourtant essentiellement pourvu, mais dont la rigidité de son apparence usuelle (sertie notamment dans des formes médiatiques standardisées) accable souvent les possibilités de reconnaissance.

11 Les caractères inouïs et inusités des interventions artistiques, dans ce contexte, reposent sur cette virtualité que se reconnaissent alors les individus de pouvoir intervenir de façon différente dans l'espace public et dans la ville, au nom de leur vision créatrice. Mais cette prérogative ne leur appartient pas en propre ou entièrement, puisqu'ils ne font la plupart du temps qu'entrevoir comment on peut se pencher, de manière attentive, sur les choses qui sont là, sur des activités qui ont cours, sur des individus et des structures qui habitent déjà l'espace urbain, mais vis-à-vis desquels l'attention ne se porte pas. Tout appartient ici en fait à la reconnaissance d'une capacité créatrice étendue, mais étendue justement à 
une concentration à l'égard des «façons de faire » - et dont l'expression artistique, une fois qu'elle les a reconnues, transforme au travers d'autres "façons de faire", susceptibles alors de rejoindre d'autre horizons que ceux des premières. Mais qu'est-ce donc qui fait que de telles déambulations ordinaires dans la ville ne paraissent " exceptionnelles" que par le détour qui les mène pour ainsi dire à l'écart des représentations usuelles de la ville contemporaine, soit au niveau des pratiques artistiques, soit des relevés « scientifiques » comme en produit la sociologie, l'urbanisme ou l'ethnologie?

Le fait de vivre dans un monde symbolique, c'est-à-dire dans un monde que nous ne comprenons et ne pouvons aborder qu'à partir des formes de représentation qui lui donnent sens, nous place dans une situation où, comme le dirait Ernst Cassirer, la représentation s'appose comme un voile sur l'environnement, sur les espaces physiques et relationnels, mais constitue pourtant simultanément la seule façon dont nous disposons pour les rendre perceptibles, sensibles et concevables, bref, de les rendre praticables. En ce sens, toutes les formes symboliques, toutes les représentations cachent et révèlent en même temps, tout en structurant le monde de relations spéculaires, convergentes, parallèles ou contradictoires. L'imaginaire symbolique qui enveloppe l'espace urbain, loin de constituer une représentation simple et univoque, construit plutôt un espace complexe et multivoque au sein duquel circulent une multitude de représentations. Il s'agit dans ce sens finalement d'un espace sémiotique très dense au sein duquel s'instituent toutes sortes de jeux entre les représentations, dont fait assurément partie une agonistique politique visant aussi une certaine hégémonie - celleci conférant par exemple à " une ville » son identité, censée alors se poser comme une définition du «bien commun » qu'offre cette ville. Cette identité demeure alors toujours une réalité mouvante, il va sans dire, relativement instable et certainement susceptible d'évolutions liées à la vie urbaine ainsi qu'au monde plus large dans lequel évolue une ville. L'imaginaire urbain tel que nous le définissons aujourd'hui apparaît donc se rapprocher davantage d'une structure dynamique et complexe de représentations multiples, mais gardant néanmoins la fonction d'être le milieu effectif de vie et de pratique du monde environnant, que la ville parvient à condenser en elle puis à faire aussi se réverbérer hors d'elle - jouant ainsi de son pouvoir d'attraction et de sa force d'influence, comme le remarquait déjà Simmel au début du XXe siècle dans sa définition de la métropole contemporaine ${ }^{12}$.

13 Loin de constituer en une détermination complète, totale et surtout fermée de la pratique, l'imaginaire symbolique de la ville se présente toujours cependant comme une contrainte, dont l'autorité est à la fois physique et représentationnelle, et au sein duquel les habitants ont à manœuvrer. L'identité d'une ville que veut représenter son imaginaire symbolique génère ainsi moins une adhésion que des attaches ou des exclusions, ainsi que de l'indifférence, tant il est vrai qu'est « ressentie » l'identité d'une ville au travers d'un spectre très large et varié d'expériences et d'évaluations. On voit alors que la marge de manœuvre qui se définit au travers de l'identité d'une ville concorde avec un certain espace herméneutique, comme lieu d'interprétation que créent inévitablement les médiations entre l'imaginaire symbolique qu'elles révèlent et les individus pour lesquels elles prennent forme. Et on voit également que ces manœuvres sont concrètement porteuses de multiples moments de libertés et d'autonomie individuelles, tout autant que d'obligation et d'hétéronomie, d'expressions comme de recherches de la personnalité, 
rendues possibles et même nécessaires par un fond d'impersonnalité que recèlent des identités collectives, générique et anonymes.

Ainsi, l'espace de dérive qui se déploie dans les coulisses de l'imaginaire collectif officiel de la ville et qui paraît lui échapper entièrement ne lui échappe finalement qu'en partie, en ce sens que, dans le cadre de la pratique immédiate de la ville par ses habitants, cet écart demeure défini par rapport au foyer de représentations instituées et officielles, tout en étant travaillé par ce qui excède la ville, et cela dans tous les sens du terme, c'est-à-dire autant dans l'interprétation que l'on en donne que dans ce qu'elle ne peut contenir en elle seule dans ses limites physiques. Par conséquent, les circulations inusitées et marginales dans la ville ne se vivent au final qu'au travers des règles lexicales et syntaxiques de son imaginaire ambiant, mais tel que celui-ci puise à un horizon pratiquement infini de possibles transformations. Les entorses et libertés prises face à ce langage, comme face à d'autres représentations instituées, ne sont alors pas ressenties comme étant exceptionnelles tant « le style » propre, l'« art de faire » ou de parler privé des individus est défini dans la relation constitutive, qu'elle soit sereine ou conflictuelle, entretenue avec l'expression ou, plus généralement, avec les représentations publiques, si complexes soient-elles ${ }^{13}$. La plasticité de l'espace public (et des pratiques privées qui le meublent et le traversent), bien qu'elle se réalise en tout temps, n'est surtout visible et audible en tant que telle, c'est-à-dire non en tant que quotidienneté mais en tant que potentiel de libération face aux représentations instituées, en tant que marginalité donc, que du moment où elle est traduite autrement, présentée d'une façon autre, dans un médium parfois alternatif, une structure perceptive ou syntaxique différente, que celle-ci soit artistique ou scientifique par exemple. C'est seulement ainsi, en reconnaissant d'abord une "forme " ou une représentation usuelle comme centre à partir duquel s'organisent les pratiques de multiples façons, ce qui ne se réalise en quelque sorte qu'en la confrontant à d'autres ayant une structure et un langage différents, que l'espace herméneutique dans lequel ces pratiques prennent place devient espace non plus immédiat, mais médiatisé, plurimédiatisé, et qu'il apparaît de là comme nouveau champ exceptionnel de possibilités pour l'interprétation.

La capacité de varier et de transformer les représentations pour un même état de chose, dans le cas qui nous intéresse ici, pour des activités qui ont cours dans la ville, comporte donc la possibilité de développer ce que Cassirer, toujours, appelait une plus grande distance critique face aux représentations qui constituent notre milieu de vie, une plus grande différenciation entre ce qu'elles sont comme forme et ce que sont les contenus qu'elles médiatisent. On peut noter que cet espace critique face aux représentations, ou à un espace de représentations en lutte, peut être reconnu comme ayant une importance capitale pour le déploiement dynamique des cultures et des styles de vie. Ce déploiement constant, qui est la consistance dynamique de la culture aux yeux de Cassirer, est avant toutes choses le résultat d'un jeu de forces entre des dimensions "mythiques » ou "représentatives », des représentations qui présentent avec une évidence moins grande leur différence face aux contenus qu'elles révèlent et cachent à la fois, qui se présentent donc avec une certaine prétention à l'absolu, et des dimensions "critiques", des représentations qui n'ont d'autres fonctions que de rendre évidentes et conscientes le caractère imparfait, différencié et autonome de la médiation face à son ambivalence. Sans s'articuler seulement à des pratiques expressives qui demandent une stricte connaissance des «règles" de la communication (au sens par exemple que Habermas prête aux " compétences communicationnelles» dans sa conception de l'espace public, dont il 
remodèle les exigences modernes en recettes contemporaines), ou une adhérence à des orientations normatives étroites, c'est plutôt d'une ouverture à l'interprétation dont il s'agit, ainsi que d'une reconnaissance de l'indétermination relative des formes dans lesquelles l'expression peut se présenter qui sont ici requises ${ }^{14}$.

On peut donc voir que la constitution dynamique et vivante d'un imaginaire de la ville se déroule ainsi à la croisée de plus d'un axe dialectique. D'abord, la dialectique entre des dimensions vernaculaires et spectaculaires définit une structure momentanée de lutte pour l'hégémonie d'une représentation totalisante et immédiate de la ville et de son identité; ensuite, cette structure entre en relation avec des structures d'« interprétation ", de "traduction", de "révélation» autres des pratiques qu'elle médiatise, qui tendent à la relativiser et à donner une plus grande légitimité à ce qu'elle comporte comme possibilités d'écart expressif. Il s'agit de reconnaître le jeu des structures expressives qui naîssent à l'intérieur de l'espace herméneutique ou de manœuvre que portent les représentations, tel qu'il apparaît comme sens possible parmi d'autres, de médiation non globale mais participant tout de même de l'imaginaire urbain qui se déploie à la mesure de ce que l'espace public permet d'accueillir en lui, pour ainsi dire à la croisée de ces deux axes dialectiques.

L'exemple de l'exposition-événement Espace mobile nous montre que la variation des représentations joue sur ces deux axes dialectiques, en instaurant par le fait même une médiation symbolique d'un ordre et d'une qualité tout autre que celle qui motive le projet du Quartier des spectacles. C'est que les dérives proposées dans chaque cas, si elles ont le même point d'origine, n'ont pas la même destination. Et c'est à ce moment que l'on peut reprendre le questionnement, peut-être plus général, sur la place et la situation des rapports entre l'expression artistique et l'expression citadine dans le contexte contemporain. Le sens de la dérive des villes est lié aussi à l'exploration à la quelle consentent la réflexion et la vie contemporaines.

\section{...aux rapports entre esthétique et éthique}

Tout le mouvement de la modernité esthétique a accompagné le développement urbain contemporain en travaillant au corps à corps l'espace public offert par la ville, mais à partir de ses marges, en fonction de cette frange de marginalité qui, toujours en opposition au centre apparent d'intérêt que suscite l'expérience citadine, entraîne une dialectique culturelle à l'image d'une dynamique véritable du politique; la figure du flâneur chez Baudelaire provoque bien entendu le bourgeois affairé; Getrude Stein, en femme de pouvoir du cercle des avant-gardes états-uniennes à Paris, déstabilise l'establishement masculin; Warhol en pape de l'underground new yorkais lutte contre les stéréotypes qui affectent les marginaux (gais, drogués, incultes) ${ }^{15}$. Toutes les avant-gardes artistiques ont voulu, depuis le milieu du XIX ${ }^{\mathrm{e}}$ siècle au moins, prendre d'assaut la ville, en inversant très exactement le sens de la "guerre culturelle » qu'elles livraient pour une transformation de la société en auto-transformation des pratiques. Qu'est-ce à dire? D'abord, que les pratiques artistiques évoluent dans le sens d'un travail de l'affection, de la perception et de la conception qui n'a d'autres finalités que cet auto-déploiement de lui-même (mouvement qu'on a souvent traduit par la formulation de "l'art pour l'art », mais sans toujours considérer la signification épistémique profonde de cette formulation pour la compréhension du rapport nécessaire des pratiques artistiques à leur extériorité); ensuite, que ce mouvement ne peut se traduire qu'au sein même d'une culture qui se voit, 
par là, toujours entrainée au-delà des limites qu'elle se fixe; également, que toute transformation des significations ne peut s'exercer que de manière auto-réflexive et endogène, afin de nourrir éventuellement une réflexion extérieure; enfin, que l'expression artistique dans ce contexte ne rencontre inévitablement ses propres limites que dans le rapport à l'espace public, réceptacle plastique de l'expression, et cela dans la mesure même où cette expression est susceptible d'en indiquer les repères et les pôles extrêmes. Et, bien que le rapport historique des avant-gardes artistiques à la société ait été aujourd'hui institutionnalisé, soit intégré officiellement dans les formations académiques des artistes (au sein des conservatoires et des universités notamment, où son enseignés les œuvres, travaux et conceptions des avant-gardes, inscrits dès lors dans notre tradition culturelle), l'art continue de jouer ce rôle dans la frange de marginalité qui est la sienne, en établissant sans relâche cette relation de tension à l'égard des aspects «centraux » de la gouverne politique et de ses directions spécifiques - en accentuant par là, si possible, son "excentricité », précisément dans la volonté de distendre cette relation dialectique avec l'espace/temps dans lequel il évolue. Dire que l'art continue de jouer ici un rôle politique à l'intérieur de la cité, c'est avancer qu'il élargit dans un sens la résonance du politique à ses dimensions non seulement «logiques " et "rhétoriques", mais bien aussi «perceptives» et «pathiques»; ainsi, la mobilisation des affects et des percepts intervient à la face des "concepts " et des "idéologèmes " censés diriger l'orientation d'ensemble du politique, de façon à les faire "résoner " (et "raisonner ») autrement. Dans un autre sens, le rôle politique de l'art se comprend alors dans la condensation de l'expression à laquelle il parvient au travers de ses formes mêmes, qui ne peuvent ainsi jamais s'intégrer « immédiatement » ni aux pratiques usuelles, ni non plus aux pratiques officielles, qu'en fonction du détour qu'elles (leur) proposent. Les pratiques artistiques sont des œuvres de médiation intrinsèques, et c'est au sein de l'espace public dans lequel elles s'insèrent qu'elles permettent la reconnaissance des autres instances de médiation qu'elles côtoient - autant celles du pouvoir que celles de l'opinion publique, ou que celles de l'individualité.

19 C'est donc d'une toute nouvelle connotation que se pare dans ce contexte le rapport entre esthétique et éthique au sein de la cité ; il y a interférence entre les deux, car leurs visées sont distinctes et ne peuvent pas être confondues, sous peine justement de ramener l'expression artistique, dans le cas qui nous occupe, aux vues du spectaculaire urbain, ou à l'inverse, de le rendre identique au vernaculaire de l'éthos collectif ${ }^{16}$. Le jeu de l'affrontement que l'art entretient vis-à-vis de l'espace public, particulièrement dans le contexte des villes contemporaines, se structure dans ce rapport agonistique que soutient sa propre expression, animée d'une relation d'opposition plus ou moins forte au politique - mais toujours dans la distance qui la sépare et la distingue de ce dernier. L'extériorité relative que l'art maintient vis-à-vis de la cité, jusqu'à sa possible excentricité, lui permet d'élargir les perspectives, au travers des émotions et des perceptions, d'un éthos urbain autrement ceinturé par le pouvoir politique à qui rien ne peut échapper, sinon des visées plus ou moins avouées d'intérêts spécifiques, mais dont les motifs mêmes trahissent cependant l'étroitesse des vues dans lesquelles il se tient la plupart du temps. Espace mobile a ainsi fait exister, le temps d'une exposition-événement, une vision autre du Quartier des spectacles montréalais, et c'est aux relais présents également dans l'espace public, aux autres formes et aux autres types de médiation, de participer à partir de là à leur propre façon à la reconnaissance des enjeux présents dans la (re)constitution du milieu urbain en cours présentement ${ }^{17}$. Le fait d'ailleurs que cette 
expérience puisse être transposée dans d'autres contextes dans le monde, ou que des artistes "étrangers» (i.e. en provenance de France ou de Belgique) aient pu intervenir directement dans cette initiative locale montréalaise, montre aussi que le rapport entre esthétique et éthique s'est affranchi des barrières nationales, et qu'il évolue dans le cadre « universel» de la ville, et au travers d'un espace public transfrontalier, qu'on peut qualifier pour cette raison de "cosmopolitique ». La ville contemporaine apparait bien comme un condensé du monde, et la reconnaissance des formes symboliques qui la constituent en propre, au sein même de la dialectique que ces dernières mettent en œuvre, affiche la profondeur d'une expérience universelle, saisie dans ses différents éclats. Aujourd'hui, partout dans le monde en effet se rencontre, avec plus ou moins d'intensité, ce type d'expérience qui permet de confronter des logiques appartenant soit au courant du tourisme de masse mondial, soit à des préoccupations de développements urbains liés à un lieu spécifique par des intérêts divers, soit aux expériences quotidiennes des individus dans leur habitat urbain, avec les expressions artistiques réfléchissant les divers aspects et enjeux de ces dynamiques.

\section{Conclusion : l'art dans la ville, aux marges du politique}

20 Nous conclurons brièvement sur cette question de la participation esthétique au politique, en insistant sur le caractère marginal de l'expression et sur son rôle de régénération de l'espace public. Indéniablement, la présence d'une exposition-événement comme Espace mobile a permis l'expression de formes esthétiques qui se sont manifestées dans un contexte politique de transformation urbaine, en jouant de manière critique et réflexive sur la signification donnée au projet du Quartier des spectacles; de cette manière, Espace mobile a introduit dans l'espace public une diversité d'expressions artistiques susceptibles d'entrer en interférence avec la vision «spectaculaire » du devenir présumé de Montréal, en tablant sur une attention à l'égard du «vernaculaire » local, mais en transposant tout autant celui-ci dans des expressions inouïes. Le débat « politique » en at-il été pour autant transformé ?

21 L'évaluation que l'on ferait en répondant à cette question tient essentiellement à la définition que l'on donne au concept de " politique ». Si l'on s'en tient au strict niveau des politiques municipales, rien ou presque en apparence ne trouble l'évolution du projet du Quartier des spectacles à Montréal, qui continue de faire son chemin au travers d'une planification partielle et d'avancées certaines (sur le plan immobilier notamment) - mais ceci occupe pour ainsi dire le devant de la scène. Et nous avons insisté sur la marginalité de l'expression artistique dans ce contexte, par rapport à cette "centralité ». Deux choses sont alors à souligner, pour caractériser ce rapport : cette marginalité expressive élargit justement le domaine de l'espace public, qui pourrait très bien, en somme, se contenter d'une façade spectaculaire comme mesure de son étendue (mais celle-ci resterait alors tronquée, voire peut-être complètement absorbée dans cette façade) ${ }^{18}$; cette marginalité expressive approfondit tout aussi bien le domaine de l'espace public, en inscrivant entre autres la conscience historique des transformations urbaines en cours dans une perspective qui attire l'attention sur la tradition du vernaculaire, en ce que cette dernière a sédimenté au fil de son évolution une histoire de la ville qui se dit simplement au travers des vestiges de sa présence.

22 La Galerie VOX a d'ailleurs pris acte de ces deux dimensions correlatives à l'expositionévénement Espace mobile: d'une part, tout une série de discussions a été développée avec 
divers habitants et intervenants du quartier pendant la réalisation des œuvres artistiques, qui demandaient souvent une "documentation vivante ", et des séminaires avec des universitaires sur le thème de l'exposition, tenus dans le cours de son déroulement, de même que des activités pédagogiques avec des étudiants de niveau secondaire, ont étendu les préoccupations artistiques à d'autres aires de la réflexion; d'autre part, tout le travail réalisé par les artistes demeure, archivé en quelque sorte sous diverses formes comme une activité témoignant des transformations en cours au sein du quadrilatère devenant granduellement le Quartier des spectacles, transformant en quelque sorte l'immédiateté de la réalisation en mémoire de cette manifestation, elle-même devenue par moments mémoire de ce que fut et de ce qu'est le quartier au moment où il devient autre chose que ce qu'il est et ce qu'il était ${ }^{19}$. Les deux axes dialectiques du développement de l'espace public ont donc été mobilisés dans ce contexte afin de régénérer la plasticité qui est la sienne.

Cette plasticité de l'espace public, sur laquelle nous avons insisté à quelques reprises ici, doit alors être entendue avant tout dans son sens esthétique. Elle tient à la capacité d'accueil des expressions, dans leurs caractères polymorphes, qui sont susceptibles d'apparaître au travers de réalisations artistiques, mais qui tiennent autant aux limites de ce que produit l'imaginaire symbolique de l'expérience urbaine contemporaine. Les formes de cette expérience, dans le travail de la signification et du sens qu'elles entretiennent, dans l'élargissement et l'approfondissement des résonances qu'elles appellent, sont susceptibles de fournir aux individus des villes du monde d'aujourd'hui des éléments de (re)composition du bien commun, comme une façon de retrouver l'hospitalité des lieux qu'ils habitent en les cultivant de diverses manières.

\section{BIBLIOGRAPHIE}

Bélanger, A., (2005), « Montréal vernaculaire/Montréal spectaculaire : dialectique de l'imaginaire urbain », Sociologie et sociétés, vol. XXXVII, n 1, pp. 13-34.

Benjamin, W., (2000), « Sur quelque thèmes baudelairiens », dans CEuvres, III, trad. M. De Gandillac, R. Rochlitz et P. Rusch, Paris, Gallimard, pp. 329-390.

Cassirer, E., (1972), La philosophie des formes symboliques, t. 2. La pensée mythique, trad. J. Lacoste, Paris, Minuit.

Cassirer, E., (1973), Le mythe de l'État, trad. B. Vergely, Paris, Gallimard.

Côté, J.-F., (2005), « Le spectacle du monde. Nouvelles formes du cosmopolitisme et espacestemps fracturés des métropoles ", Sociologie et sociétés, vol. XXXVII, nº 1, pp. 231-260.

Côté, J.-F., (2003), Le triangle d'Hermès. Poe, Stein, Warhol, figures de la modernité esthétique, Bruxelles, La Lettre Volée.

De Certeau, M., Giard, L. et Mayol, P., (1994), L'invention du quotidien. 2. Habiter, cuisiner, Paris, Gallimard.

Habermas, J., (1988), L'espace public, trad. M. B. de Launay, Paris, Payot. 
Joseph, I., (1998), La ville sans qualités, Paris, Éditions de l'Aube.

Simmel, G., (1987), Philosophie de l'argent, trad. S. Cornille et P. Ivernel, Paris, P.U.F.

Simmel, G., (1989), Philosophie de la modernité, trad. J. L. Vieillard-Baron, Paris, 2004.

Sorkin, M. (ed.), (1992), Variations on a Theme Park. The New American City and the End of Public Space, New York, Hill and Wang.

Zukin, S., (1995), The Culture of Cities, Cambridge, Blackwell.

\section{NOTES}

1. Cet article participe d'une recherche entamée sous les auspices du projet Culture of Cities (2000-2005), financé par le Conseil de recherche en sciences humaines du Canada (CRSH), et qui se poursuit présentement, sous des paramètres légèrement modifiés, dans le cadre du projet Médialité urbaine/Urban mediality (2008-2010), financé par le Fond québécois de recherche sur la société et la culture (FQRSC). Au sujet des thématiques du « spectacle du monde » et du rapport entre le «spectaculaire» et le "vernaculaire» urbains, nous renvoyons aux textes de JeanFrançois Côté (2005) et d'Anouk Bélanger (2005) du numéro de la revue Sociologie et sociétés, vol. XXXVII, $\mathrm{n}^{\circ} 1$, printemps 2005, qu'ils ont co-dirigé.

2. Le Partenariat du Quartier des spectacles est un organisme à but non lucratif, créé en 2003, et chargé de coordonner les différentes phases de transformations de ce secteur; en cela, il «...développe et met en place des projets collectifs pour renforcer la cohésion de ce milieu de vie et de création, et vise à le positionner comme un des pôles majeurs de Montréal, destination culturelle internationale». Voir à ce sujet la section "À propos de nous", sur le site Web: www.quartierdesspectacles.com. Pour donner une idée de l'orientation du projet du Quartier des spectacles, rappelons que Charles Lapointe, président-directeur-général de l'organisme Tourisme Montréal, a également été pendant un certain temps directeur général du Partenariat du Quartier des spectacles.

3. Ce quartier, dont le cœur est formé par l'angle des rues Saint-Laurent et Sainte-Catherine, a en effet longtemps été le cœur des activités liées à la prostitution à Montréal, caractérisées par les bordels qui affichaient une lumière rouge à l'extérieur comme signe distinctif de leur présence - et de là l'expression « Red light » comme désignation générique et populaire du quartier. Une des initiatives du projet du Quartier des spectacles est de reprendre, mais évidemment sous une forme complètement différente, l'idée d'une éclairage utilisant la "lumière rouge » comme indice de démarcation de son périmètre d'exploitation.

4. La présentation de l'exposition Espace mobile s'établissait au départ comme suit: «L'hypermobilité des individus aujourd'hui, le développement urbain de Montréal en archipel spécialisé, le tourisme de masse et, par conséquent, la multiplication d'activités culturelles et festives transformeront les usages du centre-ville et les manières d'y circuler. Espace mobile souhaite observer et interroger les transformations imminentes de ce territoire où VOX s'est implanté en 2004 ». La Galerie VOX, dont le mandat original couvre essentiellement le domaine de la photographie, existe depuis plus de 20 ans, et après avoir occupé différents lieux, elle s'est maintenant établie sur la rue Saint-Laurent, en plein cœur du quadrilatère visé par l'initiative du Quartier des spectacles (voir le site Web: www.voxphoto.com). Les motifs de l'exposition Espace mobile en lien avec ce projet de transformation du quartier étaient annoncés comme suit : "Sept artistes ont été invités à réaliser une série de visites de cet environnement urbain pour proposer ensuite au public de multiples parcours en faisant de l'intersection du boulevard Saint-Laurent et de la rue Sainte-Catherine l'épicentre de leurs recherches. Loin de se cantonner dans la seule transcription visuelle de la géographie physique ou urbaine de ce territoire, les parcours guidés 
ausculteront des strates variées de ce milieu - aussi bien l'espace sonore ou les représentations touristiques que l'embourgeoisement ou les équipements de surveillance, entre autres. Adoptant pour la plupart la modalité du work in progress, les projets se déploieront durant toute la durée de l'événement et prendront la forme de cartes et de propositions de trajets, de documentation photographique, sonore ou vidéo, de notations, voire de véritables circuits commentés. Ainsi la carte se superposera-t-elle concrètement au territoire, en donnant lieu à des interventions tels des échantillonnages, des rencontres, des investigations et des randonnées. La formule activera aussi un processus de va-et-vient entre la galerie et le milieu urbain, entre la documentation et l'expérience vive de la cité. Espace mobile nomme justement cette nécessité pour les artistes et le public de circuler entre l'espace de l'art et l'espace de la ville ». Marie-Josée Jean, Patrice Loubier, «Les mobiles de la manifestation », VOX Image Contemporaine, $n^{\circ} 26$, mars 2008, sans pagination.

5. James Partaik, Catalogue de présentation, VOX Image Contemporaine, $\mathrm{n}^{\circ} 26$, mars 2008 , sans pagination. Pour des informations supplémentaires sur les projets antérieurs de Partaik et sur son parcours artistique, voir notamment son site Web personnel: www3.sympatico.ca/ jamespartaik/.

6. Syn- Jean-Maxim Dufresne, Luc Lévesque, Jean-François Prost, Catalogue de présentation, VOX Image Contemporaine, $\mathrm{n}^{\circ} 26$, mars 2008 , sans pagination. Pour des informations supplémentaires sur les projets de Syn- voir notamment leur site Web officiel : http://www.amarrages.com/.

7. Il faut relever ici que le projet du Quartier des spectacles, dans la foulée de la promotion urbanistique du quadrilatère visé, influence certainement le développement immobilier dans le sens de la gentrification, et donc du déplacement physique des populations suscité par la hausse des loyers, notamment; or, si le projet prête aussi officiellement une attention à l'expression artistique «marginale» ou "émergente», cette attention demeure justement elle-même marginalisée par rapport à l'intérêt beaucoup plus prononcé qu'il porte aux initiatives plus spectaculaires et plus rémunératrices des industries culturelles (festivals, salles de spectacles, etc.). C'est dans ce sens donc qu'intervient une marginalisation de certaines couches de population et de certaines franges d'expressions dans le contexte de développement du Quartier des spectacles, qui prend alors une signification plus univoque.

8. Renaud Auguste-Dormeuil, Catalogue de présentation, VOX Image Contemporaine, $\mathrm{n}^{\circ} 26$, mars 2008, sans pagination.

9. Voir à ce sujet le Catalogue de présentation d'Espace mobile, op.cit., de même que les sites suivants, pour Jocelyn Robert (http://www.actuellecd.com/fr/bio/robert_jo/); pour Gilbert Boyer (www.gilbertboyer.com/); pour Christoph Fink (www.manifesta.org/manifesta4/en/ projects/artist71.html); pour Felicity Tayler (www.pictorialpropaganda.ca).

10. Cette reconnaissance se fait d'ailleurs à plusieurs niveaux, puisque toute la dimension «documentaire» des différents projets artistiques développés acquiert une dimension " archivistique ", dans la conservation qui en est faite par la Galerie VOX, ainsi que par le biais des différents sites Web impliqués diversement par l'exposition - nous reviendrons dans la conclusion sur cet aspect des choses, qui prend un relief particulier dans le contexte de la transformation du quartier, étant donné qu'il récupère certaines des expressions vouées à disparaître dans le processus en cours.

11. Nous tirons ces remarques des propos tenus par Marie-Josée Jean, commissaire avec Patrice Loubier de l'exposition Espace mobile, et directrice de la Galerie VOX, lors d'une entrevue réalisée avec elle le 19 août 2008.

12. Georg Simmel, «Les grandes villes et la vie de l'esprit», dans Philosophie de la modernité, trad. J. L. Vieillard-Baron, Paris, Payot, 2004 (1989), pp. 169-183. La notion de «milieu de vie » doit être comprise ici au sens que Uexkull, entre autres, donne à ce mot en biologie, soit en fonction d'une approche "écologique", mais qui prend dans ce contexte symbolique une signification culturelle. 
13. Comme l'écrit Simmel : «L'individu ne ressentira pas [...] dans sa langue maternelle, tant qu'il la parle naïvement, de lois objectives auxquelles il devrait se référer, comme si elles existaient au-delà de son propre sujet, pour leur emprunter une traduction possible de son intériorité qui soit conforme à des normes indépendantes. Au contraire, dans ce cas, l'expression se confond avec la chose exprimée ». Georg Simmel, Philosophie de l'argent, trad. S. Cornille et P. Ivernel, Paris, P.U.F., 1987, p. 592.

14. On peut relever justement ici que la dimension esthétique représente sans aucun doute la grande absente de la théorisation de l'espace public qu'a proposée Jürgen Habermas. En s'en tenant essentiellement aux discussions critiques qui entourent la production esthétique comme lieu de détermination du jugement (dans une optique kantienne), Habermas réduit la portée de l'expressivité des œuvres artistiques elles-mêmes à un horizon qui ne rejoint pas le domaine éthique de la discussion politique ; nous plaidons ici pour un élargissement de cette conception, tout en admettant que la place de l'expression demeure marginale, mais au sens fort du terme, c'est-à-dire au sens où cette marginalité intervient précisément comme une délimitation de l'espace public, comme nous le montrerons dans la prochaine section.

15. Voir notamment à ce sujet Jean-François Côté, Le triangle d'Hermès. Poe, Stein, Warhol, figures de la modernité esthétique, Bruxelles, La Lettre Volée, 2003. Baudelaire, à bien des égards un pionnier de ce rapport à la ville (comme Poe qu'il admire), écrit dans une lettre : «Quel est celui de nous qui n'a pas, en ses jours d'ambition, rêvé le miracle d'une prose poétique, musicale sans rythme et sans rime, assez souple et assez heurtée pour s'adapter aux mouvements lyriques de l'âme, aux ondulations de la rêverie, aux soubresauts de la conscience ? C'est surtout dans la fréquention des villes énormes, c'est du croisement de leurs innombrables rapports que naît cet idéal obsédant ». Charles Baudelaire, cité par Walter Benjamin, "Sur quelque thèmes baudelairiens ", dans Euvres, III, trad. M. De Gandillac, R. Rochlitz et P. Rusch, Paris, Gallimard, 2000, p. 342.

16. La confusion inverse est tout aussi instructive: associer le spectaculaire urbain, ou son opposé, le vernaculaire, à des pratiques artistiques, revient à nier le caractère spécifique de ces pratiques, et la définition de l'art.

17. Lors de l'entrevue cité précédemment, la directrice de la Galerie VOX, Marie-Josée Jean, indiquait la volonté de donner suite à l'exposition dans un contexte de publication d'un ouvrage de réflexion plus général sur les questions abordées lors de l'événement, afin de lui donner justement une résonance et une pérénité plus étendues.

18. C'est cette conclusion que tirent nombre d'études sur la «disparition » ou la «fin» de l'espace public dans la vague de commercialisation qui atteint les entreprises de transformation des villes contemporaines, comme le montrent les contributions dans l'ouvrage de Michael Sorkin, Variations on a Theme Park. The New American City and the End of Public Space, New York, Hill and Wang, 1992.

19. Selon la directrice de la Galerie VOX, et co-commissaire de l'exposition Espace mobile, MarieJosée Jean, au-delà de la réception proprement médiatique de l'événement, il faut compter aussi sur la réception de la critique spécialisée, qui agit dans ce cas comme une autre instance de médiation de l'espace public, mais légèrement décalée dans le temps et dans l'espace par rapport à la manifestation; également, les suites à donner au projet, sous forme de la publication d'un ouvrage, entre autres, ajoutera à sa pérennité. Ces propos ont été recueillis lors de l'entrevue mentionnée plus haut. 


\section{RÉSUMÉS}

Cet article examine le cas de l'exposition Espace mobile présentée en 2008 par la Galerie VOX, de Montréal, qui fait figure d'exemple-type de la participation artistique dans le contexte contemporain du développement des espaces publics urbains entraînant des restructurations et des re-symbolisations. L'étude de ce cas conduit dans un premier temps à montrer comment les expressions artistiques vont à l'encontre du projet de Quartier des spectacles à Montréal, dès lors qu'elles visent à faire accéder à la représentation tout un ensemble d'expériences marginales au sein de l'espace urbain visé par ce projet. Dans un deuxième temps, quelques propositions sont avancées quant au rôle que peuvent jouer les mises en forme artistiques dans la régénération des espaces publics urbains, dans la formulation d'une interprétation plus large des rapports entre la dimension esthétique et la dimension éthique de l'expérience de la ville.

In this article, the exhibition Espace mobile presented in 2008 in Montréal by the Galerie VOX, is analyzed as an example of the artistic participation taking place in the context of urban public spaces being restructured and re-symbolized. This case study leads first, to show how artistic expressions go against the Quartier des spectacles project in Montréal, to the extent that these expressions seek to represent marginalized and invisible experiences of the urban space targeted by this project. Secondly, some propositions are made about the role that aesthetic expressions can play in regenerating urban public spaces, within the larger interpretation of the relations existing between the aesthetic and ethical dimensions of the experience of city life.

\section{INDEX}

Mots-clés : espace public, esthétique, ville, éthique, art, exposition, représentation, politique Keywords : public sphere, aesthetics, city, ethics, art, exhibition, representation

\section{AUTEURS}

\section{JEAN-FRANÇOIS CÔTÉ}

Jean-François Côté est Professeur titulaire au Département de sociologie de l'Université du Québec à Montréal. Il est chercheur associé au GIRA (Groupe inter-universitaire de recherche sur les Amériques) à l'Institut national de la recherche scientifique (INRS - Urbanisation, culture et société). Il est également membre du groupe de recherche Médialité urbaine/Urban Mediality financé par le Fonds québécois de recherche sur la société et la culture.

\section{MAUDE PUGLIESE}

Maude Pugliese est ingénieure, et termine une maîtrise en sociologie à l'Université du Québec à Montréal sur la philosophie des formes symboliques d'Ernst Cassirer. Elle est également assistante de recherche et membre du groupe de recherche Médialité urbaine/Urban Mediality. 